\title{
Effets d'une fertilisation et d'un amendement sur l'immobilisation d'éléments dans la biomasse d'un peuplement adulte d'épicéa commun (Picea abies L Karst)
}

\author{
S Belkacem, C Nys* ${ }^{\star}$ D Gelhaye
}

INRA, recherches forestières, sols forestiers, Champenoux, 54280 Seichamps, France

(Reçu le 10 septembre 1991; accepté le 5 février 1992)

\begin{abstract}
Résumé - Une fertilisation azotée et des amendements calciques ont été appliqués en 1980 dans un peuplement d'épicéa commun adulte situé dans les Ardennes françaises. La biomasse par compartiment et les éléments minéraux immobilisés ont été évalués pour chacun des traitements.

L'évaluation de la quantité de matière sèche ou d'éléments minéraux est basée sur l'établissement de tarifs, lois mathématiques établies suivant le modèle de la régression.

Deux groupes se distinguent nettement, le témoin et le traitement "Azote" d'une part avec une production moyenne de biomasse et le groupe des traitements calcaires d'autre part, pour lequel on constate un gain de biomasse d'environ $20 \%$ et une immobilisation plus importante d'éléments minéraux, due principalement à ce gain de matière sèche.

L'effet du traitement "Azote" sur l'immobilisation des éléments minéraux est peu marquée (signification à $10 \%$ ) par rapport au témoin. Par contre, les arbres ayant reçu un amendement calcique ont des teneurs en éléments minéraux plus élevées en particulier pour le calcium, dans une moindre mesure pour le magnésium, éléments qui ont été considérés au niveau du seuil de carence dans le témoin et le traitement «Azote». Des symptômes de dépérissement sont visibles sur les arbres de ces 2 derniers cas, et même accentués dans le traitement "Azote".
\end{abstract}

biomasse / minéralomassse / forêt / amendement / fertilisation / dépérissement / Picea abies = épicéa commun

Summary - Effect of fertilization and liming on the biomass and element content in a mature Norway spruce stand (Picea abies L Karst). Biomass and mineral content were evaluated in a spruce stand in the French Ardennes. The effect of nitrogen fertilization and liming with carbonate or slag was studied. The stand is a 60 -year-old Norway spruce plantation on a poor and acidic soil, with a pH of around 4 in the semi-organic horizons. Symptoms of yellowing and needle loss are present in the control plot and aggravated by the nitrogen treatment. Eight trees in each treatment were sampled 8 years after fertilization as shown in figure 1. Figure 2 shows, for the top 10 verticils, the change in the element concentration distribution related to the position along the stem and to the

\footnotetext{
* Correspondance et tirés à part
} 
treatments. The mean values of the concentration and the variance analysis are presented in table I by a group of treatments due to the fact that the nitrogen treatment is not highly different from the control, but the limed treatments (carbonate or slag) are significantly different from the group control + nitrogen fertilization. Figure 4, which depicts the results of the principal component analysis, shows the separation between the groups of limed and unlimed plots, especially in the youngest parts of the tree which have grown since the fertilization was applied (in the needles of the higher branches). The quantitative determination of the dry matter and amounts of elements has been carried out using model of regression with the $\mathrm{DBH}\left(\mathrm{C}_{130}\right)$ as the explicative variable. Table III provides some examples of the relations. These relationships applied to the tree inventory give us the values of the biomass and the element content, as summarised in table IV. The group of limed treatments is different from that of unlimed plots. Figure 4 shows the effects of the treatments for the dry matter (DM) and total mineral content in the different parts of the tree. On this site which is poor in calcium and magnesium the addition of calcium improves the health of the trees both directly and indirectly.

biomass / element content / forest decline / nitrogen / fertilization / liming / Picea abies = Norway spruce

\section{INTRODUCTION}

Au début des années 1980, une expérience de fertilisation et amendement a été mise en place dans un peuplement adulte d'épicéa commun (Picea abies L Karst) à la Croix-Scaille, région de Monthermé dans les Ardennes primaires. Son but était de remédier à une nutrition minérale déficiente.

En 1986, les mêmes symptômes de dépérissement que ceux observés en 1983 dans les Vosges (Landmann et al, 1987) (défoliation et jaunissement des aiguilles) sont apparus sur le site expérimental de la Croix-Scaille (Nys, 1989). Les effets du dépérissement diffèrent selon les traitements appliqués d'où l'intérêt d'axer une étude sur la redistribution qualitative et quantitative des éléments minéraux dans l'arbre $\epsilon t$ la conséquence sur la biomasse de ce peuplement.

Dans ce type de sol à faibles réserves en cations notamment ( $\mathrm{Mg}, \mathrm{Ca}$ ) (Nys, 1989) et où l'acidification ne cesse de croître avec les apports externes de polluants (N, S) (Nys et Ranger, 1988), les effets des amendements et de la fertilisation azotée ont été différents. Dans le traitement «Azote» $(N)$, les signes de dépéris- sement (défoliation et jaunissement des aiguilles) sont plus accentués que dans le témoin ( $T$ ) et dans les traitements "Calcaire" ( $\mathrm{Ca}, \mathrm{PCa}$ ) les arbres ont presque tous un aspect d'arbre sain (Nys, 1989). Ces états sanitaires différents ne font que révéler le déséquilibre nutritionnel de ce peuplement. Cette étude permettra de mieux cerner l'influence des apports sur la concentration des éléments minéraux majeurs dans les différentes parties de l'arbre (compartiments) en particulier de ceux qui n'ont pas été apportés par fertilisation et notamment le magnésium qui est soupçonné d'être à un niveau insuffisant dans l'écosystème.

II s'agit de mettre en évidence :

- les éléments qui font le plus défaut dans la nutrition des arbres (par le biais de l'analyse du matériel végétal; aiguilles et bois);

- l'influence de ces traitements sur la redistribution des éléments minéraux ainsi que sur la minéralomasse dans les différents compartiments de l'arbre.

Les 2 aspects, variation qualitative puis quantitative des éléments minéraux dans la biomasse forestière, seront traités. Les résultats permettront d'évaluer les différences entre peuplements dépérissants et 
sains, de comprendre l'effet d'un dépérissement sur un peuplement forestier et de trouver les moyens d'y remédier par des apports d'un amendement. L'évaluation de la minéralomasse permettra, par ailleurs, d'avoir une idée sur l'immobilisation des éléments majeurs dans la biomasse et ainsi de pouvoir prévoir la quantité d'éléments susceptible d'être perdue par l'écosystème forestier au moment des traitements sylvicoles (éclaircies, coupes, etc).

\section{MATÉRIEL ET MÉTHODES}

\section{La station}

L'étude a été réalisée au lieu dit la Croix-Scaille dans le massif forestier des Ardenres irançaises. L'altitude est de $480 \mathrm{~m}$ et le climat est de type montagnard, froid et humide. La pluviométrie annuelle moyenne est de $1300 \mathrm{~mm}$.

Le sol est un sol brun acide à humus de type moder et à faibles réserves minérales (Dubs, 1988; Nys, 1989). II est développé sur une couche de limon reposant sur des schistes du Révinien.

Le peuplement est une pessière (Picea abies $L$ Karst) issue d'une plantation de 1936 après une coupe à blanc d'un taillis-sous-futaie peu productif qui se rattachait, sur le plan phytosociologique, à une chênaie à bouleaux. En 1980, à l'implantation de l'essai, la hauteur dominante variait de 22 à $24 \mathrm{~m}$, la circonférence moyenne était alors de $83 \mathrm{~cm}$ et la surface terrière de $36,8 \mathrm{~m}^{2}$ pour un nombre de tiges de $680 / \mathrm{ha}$ (Nys, 1989). En 1984 une éclaircie a ramené ce nombre à 585 tiges/ha. Les estimations des accroissements moyens et courants par ha ont été réalisées à partir des tables de production de Dagnélie et al (1988) pour les Ardennes belges. L'accroissement moyen du peuplement est de $11,5 \mathrm{~m}^{3} \cdot \mathrm{ha}^{-1} \cdot \mathrm{an}^{-1}$ et l'accroissement courant à 50 ans est évalué à $14,6 \mathrm{~m}^{3} \cdot \mathrm{ha}^{-1} \cdot \mathrm{an}^{-1}$.

\section{Traitements de fertilisation}

- témoin $(T)$ : traitement sans engrais;

- azote (N) : $100 \mathrm{~kg} \cdot \mathrm{ha}^{-1}$ d'azote sous forme d'ammonitrate $\left(\mathrm{NH}_{4} \mathrm{NO}_{3}\right)$. L'azote a été répandu au printemps 1981 puis renouvelé au printemps 1983, soit au total $200 \mathrm{~kg} \mathrm{ha}^{-1}$;

- calcaire broyé $(\mathrm{Ca}): 2500 \mathrm{~kg} \cdot \mathrm{ha}^{-1}$ de calcaire broyé à plus de $99 \%$ de $\mathrm{CaCO}_{3}$;

- PCa (PCa) : 150 kg.ha-1 de $\mathrm{P}_{2} \mathrm{O}_{5}+2500$ kg.ha-1 de $\mathrm{CaCO}_{3}$ soit $1250 \mathrm{~kg} \cdot \mathrm{ha}^{-1}$ de scories $+1600 \mathrm{~kg} \mathrm{ha}^{-1}$ de calcaire broyé.

Les engrais $(\mathrm{Ca})$ et $(\mathrm{PCa})$ ont été épandus en plein à l'automne 1980. Les 4 traitements cidessus sont répétés 4 fois. Les placettes fertilisées ont une dimension de $50 \mathrm{~m} \times 50 \mathrm{~m}$ correspondant à la zone d'application des engrais. La distance minimale d'isolement entre zones de mesure est de $20 \mathrm{~m}$.

L'état sanitaire de ce peuplement est lié au traitement. Dans le $(T)$, les constatations faites au moment de la réalisation de cette étude montrent une relative stabilité du jaunissement et des pertes d'aiguilles. Dans le traitement $(N)$, les symptômes de dépérissement sont plus accentués. Dans les traitements $(\mathrm{Ca})$ et $(\mathrm{PCa})$, l'état sanitaire des arbres s'est nettement amélioré (Nys, 1989; Belkacem, 1990).

\section{Échantillonnage et mesure de la biomasse}

L'inventaire de tous les arbres a été effectué sur une surface de $900 \mathrm{~m}^{2}$ dans chaque placeau. Les arbres retenus pour la détermination de la biomasse sont représentatifs des classes de circonférence du peuplement.

Pour la mesure de la biomasse, 8 arbres ont été échantillonnés dans chacun des 4 traitements du bloc 1 du site expérimental. Les arbres abattus ont fait l'objet de mesures et de prélèvements de leurs différentes parties (compartiments) (fig 1). 


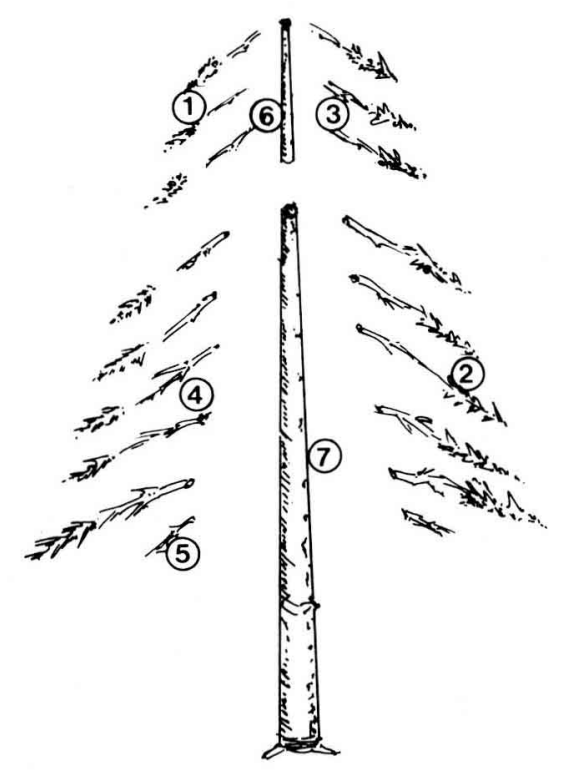

Fig 1. Schéma d'échantillonnage des compartiments de l'arbre. Dix verticilles supérieurs (1) aiguilles; (3) branches, (6) tronc. Parties inférieures (2) aiguilles; (4) branches vivantes; (5) branches mortes; (7) tronc.

Les échantillons végétaux prélevés sont séchés à l'étuve à $65^{\circ} \mathrm{C}$, broyés et homogénéisés. L'azote est déterminé par colorimétrie, sur l'Autoanalyseur II Technicon, après minéralisation en milieu sulfurique (méthode Kjeldahl modifiée) et les autres éléments ( $, P, C a, K, M g$, $\mathrm{Al}, \mathrm{Fe}$ ) sont minéralisés en milieu perchlorique puis dosés par ICP (Clément, 1977).

\section{Traitement des données}

\section{Les tarifs de biomasse, ou de minéralomasse}

Pour évaluer la biomasse et la minéralomasse, il a été tenu compte de la position des branches dans la couronne, car la teneur des tissus varie avec leur position dans l'arbre (Riedacker, 1968; Ranger, 1981; Lemoine et al, 1990).
Pour cette évaluation il s'agit d'établir, par compartiment, pour la biomasse et pour tous les éléments minéraux des relations mathématiques avec les paramètres dendrométriques. Différents paramètres sont utilisés selon les auteurs, circonférence du tronc à $1,30 \mathrm{~m}\left(C_{130}\right)$ (Rapp, 1978), diamètre des branches à $10 \mathrm{~cm}$ du tronc $\left(D_{10}\right)$ (Ranger, 1981) et circonférence à $1,30 \mathrm{~m}\left(C_{130}\right)$, hauteur et $D$ combinés $\left(D^{2}\right)$, (Satoo et Madgwick, 1982).

Différents modèles ont été testés avec ces variables ef choisis en fonction des résultats de l'étude des résidus des régressions : linéaire (Rapp, 1978), allométrique (Baskerville, 1972; Ranger et al, 1988) ou curvilinéaire (Belkacem, 1990).

\section{Analyse statistique}

Une analyse de variance multivariable à un ou plusieurs facteurs contrôlés a été appliquée aux données. Elle a permis d'évaluer l'effet des traitements sur la redistribution des éléments dans les parties épigées de l'arbre. Elle a été complétée par une analyse en composantes principales ACP (Dagnélie, 1974).

\section{RÉSULTATS}

\section{Analyse qualitative des éléments minéraux}

Les concentrations en bioéléments varient avec la position des organes dans la couronne (Wells et Metz, 1963; Ranger, 1981). II est indispensable d'en tenir compte pour apprécier la nutrition minérale des arbres (Bonneau, 1988). Les compositions moyennes des aiguilles de l'année des verticilles proches de la cime sont plus riches en $N, P$ et $K$ que celles situées dans la partie inférieure de l'arbre (Bonneau, 1986). Certains auteurs ont noté que chez les arbres à feuillage persistant, la teneur des aiguilles varie avec leur âge. D'après Hoehne (1964) la teneur, en certains éléments au moins, diminuerait d'année en 
année. Dans ce travail, les aiguilles d'un même verticille ont été regroupées avant analyse et nous étudions les 10 premiers verticilles (1980-1989) correspondant à la période post-fertilisation.

\section{Évolution de la teneur moyenne des aiguilles en éléments minéraux selon la position des verticilles}

La figure 2 montre la variation de la teneur moyenne de certains éléments en fonction de la position des aiguilles dans les premiers verticilles échantillonnés séparément.

\section{Le soufre}

La teneur en soufre varie relativement peu à partir du $6^{\mathrm{e}}$ verticille (fig 2 ). La différenciation apparaît entre la première et la cinquième année. Cette différence de variation constatée est due au fait que le vieillissement des aiguilles s'accompagne d'une stabilisation progressive de leurs concentrations (Le Goaster, 1989). Les teneurs en soufre dans les traitements (Ca) et ( $\mathrm{PCa}$ ) sont plus élevées, du moins pour les 5 premiers verticilles.

\section{Le potassium}

A partir du $6^{\mathrm{e}}$ verticille, la teneur en potassium tend à se stabiliser; toutefois dans le traitement $(N)$, ces teneurs sont plus élevées que dans le $(T)$ et les autres traitements.

\section{Le magnésium}

La concentration en magnésium diminue du premier au troisième verticille puis se stabilise à partir du quatrième verticille pour les traitements $(N),(T)$ et du septième pour le ( $\mathrm{PCa})$. La concentration en magnésium dans un peuplement non dépérissant est élevée dans toutes les classes d'âge d'aiguilles et augmente avec l'âge plus rapidement que dans un peuplement dépérissant (Oren et al, 1988). Ceci fait penser à l'existence d'une carence magnésienne dans les traitements $(T)$ et $(N)$.

\section{Le calcium}

La teneur est stable dans le (T) et le traitement $(\mathrm{N})$ mais augmente moins nettement avec l'âge des verticilles dans les traitements (Ca) et (PCa). Une forte baisse de concentration ou une trop faible augmentation pour le calcium entre première et deuxième année d'aiguilles peut être indicatrice de difficultés d'alimentation (Bonneau, 1988). C'est ce qui apparaît notamment dans le traitement $(\mathrm{N})$ avec $0,33 \%$ à $0,36 \%$ de calcium dans les 2 premiers verticilles et $0,19 \%$ dans le dixième verticille. Le calcium s'accumule préférentiellement dans les tissus âgés et plus spécialement dans les écorces (Le Goaster, 1989), mais
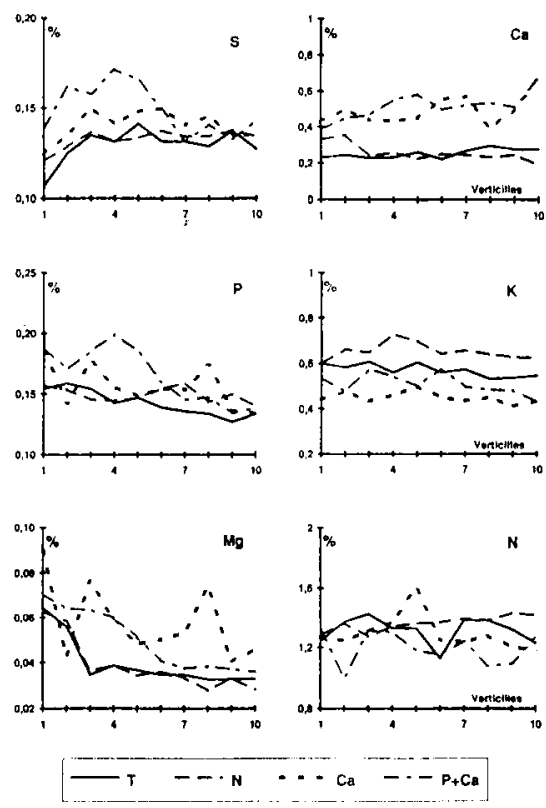

Fig 2. Modification de la concentration des aiguilles en fonction de la position du verticille. 
il n'est pas transféré depuis les aiguilles sénescentes (Oren et al, 1988).

\section{L'azote}

L'allure des courbes de la concentration selon la hauteur du verticille est indépendante du traitement. La variation de cet élément en fonction de la position des aiguilles des verticilles analysés et des traitements est apparemment anarchique et on ne peut pas tirer de conclusion.

\section{Distribution des éléments minéraux dans la biomasse}

Dans la majorité des cas, plus particulièrement dans les aiguilles des 10 premiers verticilles la concentration des éléments est significativement différente selon le type de traitement (tableau I). Deux groupes de traitements se distinguent, d'une part le $(T)$ et le traitement $(\mathrm{N})$, d'autre part les traitements $(\mathrm{Ca})$ et $(\mathrm{PCa})$. La concentration de la plupart des éléments minéraux sauf $\mathrm{N}$ et $\mathrm{K}$ est plus élevée dans le groupe de traitement (Ca) et ( $\mathrm{PCa}$ ) que dans le groupe de traitement (T) et $(\mathrm{N})$. Par contre, à l'intérieur des groupes de traitements, la concentration de presque tous les éléments minéraux n'est pas significativement différente (Belkacem, 1990). La teneur en Mg est très faible, et à un degré moindre celle de $\mathrm{Ca}$. La nutrition minérale des arbres semble déficitaire en $\mathrm{Mg}$ avec une moyenne de

Tableau I. Concentration moyenne des éléments minéraux par groupe de traitement.

\begin{tabular}{|c|c|c|c|c|c|c|c|c|}
\hline $\begin{array}{l}\text { Localisaion des éléments } \\
\text { et traitement } 1\end{array}$ & $S$ & $P$ & $F e$ & $\begin{array}{l}M n \\
(\%)\end{array}$ & $A l$ & $M g$ & $\mathrm{Ca}$ & $K$ \\
\hline
\end{tabular}

Aiguilles des 10 premiers verticilles [1]

$\begin{array}{llllllllll}\text { Groupe 1 } & 0,131 & 0,146 & 0,009 & 0,075 & 0,019 & 0,039 & 0,254 & 0,613 & 1,338 \\ \text { Test } F & 2 * * & * * & \text { NS } & * * * & * * * & * * & * * * & * \star * & * * * \\ \text { Groupe 2 } & 0,145 & 0,162 & 0,009 & 0,099 & 0,021 & 0,054 & 0,505 & 0,480 & 1,246\end{array}$

Aiguilles branches inférieures [2]

$\begin{array}{llllllllll}\text { Groupe 1 } & 0,124 & 0,123 & 0,012 & 0,074 & 0,022 & 0,032 & 0,268 & 0,590 & 1,391 \\ \text { Test } F & \text { NS } & \text { NS } & \text { NS } & \text { NS } & \text { NS } & \text { NS } & * * & \text { NS } & \text { NS } \\ \text { Groupe 2 } & 0,109 & 0,111 & 0,009 & 0,112 & 0,018 & 0,036 & 0,619 & 0,438 & 1,197\end{array}$

Bois des 10 premiers verticilles [3]

$\begin{array}{llllllllll}\text { Groupe 1 } & 0,069 & 0,105 & 0,011 & 0,040 & 0,012 & 0,046 & 0,275 & 0,519 & 0,670 \\ \text { Test } F & \text { N }^{* *} & \text { NS } & \text { NS } & * * * & \text { NS } & * * * & * * * & \text { NS } & \text { NS } \\ \text { Groupe 2 } & 0,077 & 0,107 & 0,010 & 0,035 & 0,011 & 0,052 & 0,330 & 0,487 & 0,641\end{array}$

Bois branches inférieures [4]

$\begin{array}{llllllllll}\text { Groupe 1 } & 0,059 & 0,062 & 0,011 & 0,039 & 0,012 & 0,028 & 0,291 & 0,310 & 0,515 \\ \text { Test } F & \text { NS } & * & \text { NS } & * & \text { NS } & \text { NS } & *_{* \star *}^{*} & * & * * \\ \text { Groupe 2 } & 0,060 & 0,068 & 0,015 & 0,040 & 0,015 & 0,031 & 0,380 & 0,275 & 0,610\end{array}$

Bois du tronc [7]

$\begin{array}{llllllllll}\text { Groupe 1 } & 0,014 & 0,013 & 0,001 & 0,016 & 0,002 & 0,012 & 0,108 & 0,168 & 0,120 \\ \text { Test } F & \text { NS } & \text { NS } & \text { NS } & * & * & \text { NS } & \text { NS } & \text { NS } & * * * \\ \text { Groupe 2 } & 0,015 & 0,013 & 0,001 & 0,015 & 0,002 & 0,012 & 0,121 & 0,165 & 0,138\end{array}$

1 Traitement : groupe $1(\mathrm{~T}),(\mathrm{N})$; groupe $2(\mathrm{Ca}),(\mathrm{PCa})$.

NS : non significatif; " significatif au seuil de $5 \% ;{ }^{* *}$ significatif au seuil de $1 \%$; ${ }^{* *}$ : significatif au seuil de $1 \%$. 
$0,04 \%$ dans le traitement $(N)$. La moyenne de $\mathrm{Mg}$ a augmenté sensiblement dans le traitement $(\mathrm{Ca})(0,054 \%)$. Les différences de teneurs des aiguilles et du bois des 10 premiers verticilles pour la majorité des éléments, sont hautement significatives entre groupe $1(\mathrm{~N})$ et $(\mathrm{T})$ ) et groupe 2 ( (Ca) et (PCa)). La faible teneur en magnésium traduit parfaitement la carence magnésienne observée dans des peuplements dépérissants (Oren et al, 1988) et due à la perte par le sol de quantités relativement élevées d'éléments par lessivage notamment de magnésium et de calcium (Bonneau et Landmann, 1988; Nys, 1989; Becquer et al, 1990). Oren et al (1988) ont évalué les transferts en magnésiurr. des aiguilles âgées aux aiguilles de première année à $75 \%$ dans les stations saines et $50 \%$ seulement dans les stations aux arbres dépérissants. Dans les aiguilles des 10 premiers verticilles et des branches basses, les teneurs en azote ont diminué : cela pourrait s'interpréter comme un effet négatif momentané du calcaire sur la minéralisation de l'azote (Andersson, 1989). La concentration en calcium est plus élevée dans le (T) $(0,25 \%)$ et dans le traitement $(N)(0,26 \%)$ que celle trouvée par Nys (1989) dans les mêmes traitements, $0,17 \%$ et $0,14 \%$ respectivement, mais ces teneurs restent cependant faibles par rapport au seuil optimal 0,3-0,6\% (Bonneau, 1988). Le phosphore dans l'ensemble des aiguilles du premier verticille a une teneur

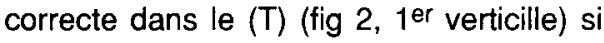
on se réfère aux normes de composition foliaire (Bonneau, 1988). Sa teneur augmente dans les traitements $(\mathrm{Ca})$ et plus encore dans le traitement (PCa). Pour les autres éléments, tels que $\mathrm{Fe}, \mathrm{Mn} \epsilon t \mathrm{Al}$, les teneurs sont convenables.

En se référant aux teneurs des éléments dans les pousses annuelles mesurées par Nys (1989), les carences en calcium et en magnésium apparaissent nettement. Ces valeurs sont respectivement pour le calcium et le magnésium de $0,17 \%$ et $0,035 \%$ dans le (T), $0,14 \%$ et $0,023 \%$ dans $(\mathrm{N}), 0,29 \%$ et $0,046 \%$ dans (Ca) et $0,21 \%$ et $0,053 \%$ dans (PCa).

L'effet des traitements est sensible dans le compartiment bois (bois + écorce) des branches des 10 premiers verticilles et l'est moins dans celui des branches inférieures (tableau I) contrairement au compartiment tronc (7). L'effet des traitements est significatif pour l'azote dans les compartiments du bois de tronc et des branches âgées. II n'y a pas d'effet sur la concentration en calcium dans le bois du tronc contrairement à ce qu'on observe pour les branches, cela peut s'expliquer par le fait qu'une grande partie du bois a été formé avant l'apport des fertilisants. II faudrait s'intéresser plutôt aux derniers cernes pour voir l'effet des traitements sur ce compartiment de l'arbre. Dans le bois des 10 premiers verticilles, la concentration de la majorité des éléments minéraux diminue ( $\mathrm{K}, \mathrm{Mg}, \mathrm{P}, \mathrm{N}$ et $\mathrm{S}$ ) et se stabilise avec l'âge (Ca).

\section{Équilibre entre éléments minéraux dans les aiguilles (pousse de l'année), relation avec l'état sanitaire des arbres}

L'équilibre entre les éléments est un indice important pour une bonne appréciation de la nutrition minérale des arbres. Autrement dit, chaque élément doit se trouver non seulement à une teneur optimale, mais ces teneurs doivent être dans des rapports convenables.

Le tableau II permet d'analyser les effets des traitements. Les valeurs calculées à partir des aiguilles de l'année en cours

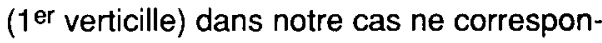
dent pas toujours aux valeurs citées par Bonneau (1986) pour des aiguilles de l'année, mais elles nous permettent de sé- 
Tableau II. Concentration des principaux éléments minéraux et quotients d'équilibre entre éléments dans les aiguilles de l'année ( $1^{\mathrm{er}}$ verticille) par traitement.

\begin{tabular}{llllll}
\hline & $T$ & $N$ & $C a$ & $P C a$ & Normes ${ }^{*}$ \\
\hline $\mathrm{S}(\%)$ & 0,11 & 0,12 & 0,12 & 0,14 & $0,12-0,18$ \\
$\mathrm{P}(\%)$ & 0,15 & 0,16 & 0,19 & 0,19 & $0,19-0,25$ \\
$\mathrm{Mg}(\%)$ & 0,06 & 0,06 & 0,09 & 0,07 & $0,1-0,14$ \\
$\mathrm{Ca}(\%)$ & 0,23 & 0,33 & 0,43 & 0,38 & $0,3-0,5$ \\
$\mathrm{~K}(\%)$ & 0,6 & 0,6 & 0,44 & 0,54 & $0,6-0,8$ \\
$\mathrm{~N}(\%)$ & 1,24 & 1,28 & 1,26 & 1,32 & $1,5-1,9$ \\
$\mathrm{~N} / \mathrm{P}$ & 8,3 & 8 & 6,6 & 6,9 & $10-15$ \\
$\mathrm{~N} / \mathrm{Mg}$ & 19,3 & 20,3 & 14 & 18,6 & $<17,5$ \\
$\mathrm{~K} / \mathrm{Ca}$ & 2,6 & 1,88 & 1,02 & 1,42 & 1,3 \\
$\mathrm{~S} / \mathrm{N}$ & 0,085 & 0,094 & 0,098 & 0,1 & 0,063 \\
& & & & & \\
\hline
\end{tabular}

*Bonneau, 1988.

parer les traitements en fonction de leur état sanitaire. Ces valeurs peuvent être révélatrices d'un déséquilibre entre les éléments, notamment $\mathrm{N} / \mathrm{Mg}$, à cause de la faible teneur en magnésium dans les traitements $(T)$ et $(N)$. Les valeurs de N/P sont normales dans le $(T)$ et le traitement (N) mais trop faibles dans les traitements (Ca) et ( $\mathrm{PCa}$ ) probablement à cause d'une insuffisance d'alimentation azotée due à un effet négatif momentané du calcaire. On remarque aussi que le rapport $\mathrm{S} / \mathrm{N}$ est plutôt élevé par rapport à la normale, ce qui peut traduire un déficit en azote, mais il faudrait analyser le soufre soluble pour confirmer l'insuffisance de la nutrition azotée.

La teneur des aiguilles en potassium et en calcium peut traduire un déséquilibre nutritionnel dans les traitements (Ca) et (PCa) : la teneur en calcium $(0,25 \%)$ est faible et celle en potassium $(0,57 \%)$ est élevée dans le (T) tandis que dans le traitement $(\mathrm{Ca})$, la teneur en calcium $(0,43 \%)$ augmente et celle en potassium $(0,44 \%)$ diminue. La teneur en magnésium suit la même évolution que celle du calcium. $\mathrm{Ce}$ phénomène pourraît probablement s'expliquer par la diminution des réserves de calcium et de magnésium dans le sol. L'arbre compense en absorbant, en valeur relative, plus de potassium, lequel se trouve en quantité suffisante dans le sol.

\section{Les modèles d'évaluation de la biomasse}

L'évaluation de la biomasse et du contenu en éléments minéraux (minéralomasse) des arbres et du peuplement est obtenue en appliquant des relations établies à partir des mesures et analyses des arbres échantillons. Elles sont ensuite appliquées à l'inventaire du ou des peuplements. Ces relations dépendent du compartiment, de l'élément et du traitement de fertilisation, mais certaines lois générales se dégagent. 


\section{Les tarifs et le calcul de la biomasse et de la minéralomasse}

II s'agit d'établir pour la biomasse et pour tous les éléments minéraux des relations mathématiques avec les paramètres dendrométriques.

L'étude des liaisons entre la concentration minérale et les paramètres dendrométriques $D_{10}$ ou $C_{130}$ montre 3 possibilités permettant l'évaluation de la biomasse et de la minéralomasse : absence de liaison, liaison linéaire ou curvilinéaire (Belkacem, 1990). Ces relations se rapprochent de celles trouvées par Nys et al (1983) pour les compartiments aiguilles et branches d'épicéa.

En l'absence de liaison, la biomasse ou la minéralomasse du compartiment (exemple du $\mathrm{Ca}, \mathrm{Mn}$ des aiguilles des branches inférieures, de la minéralomasse de la cime et des branches mortes) sont assimilées à la moyenne de concentration pondérée à la matière sèche.

S'il y a liaison linéaire, l'évaluation de la minéralomasse ou de la biomasse (par exemple la matière sèche des aiguilles des branches inférieures et $\mathrm{S}, \mathrm{P}, \mathrm{Al}, \mathrm{Zn}, \mathrm{Mg}, \mathrm{K}$, $N)$ résulte d'une relation de la forme $: Y=a$ $+b X(Y=$ masse de matière sèche; $X=$ diamètre $D_{10}$ ou circonférence $C_{130}$ ).

Les constantes $a$ et $b$ sont calculées à partir des arbres échantillons par le modèle de régression. Ce modèle mathématique simple est utilisé dans le cas des aiguilles et du bois des branches.

Dans le cas du bois du tronc, le modèle dit allométrique (Baskerville, 1972) est utilisé pour calculer la minéralomasse $(S, P$, $\mathrm{Al}, \mathrm{Mg}, \mathrm{N})$. C'est en fait un modèle linéaire en échelle logarithmique qui exprime une proportionnalité entre un accroissement relatif de 2 parties de l'arbre (Pardé, 1980). II est de la forme $\log (Y)=a+b \log (X)$ et les variables $(X, Y)$ ainsi que les cons- tantes ( $\mathrm{a}$ et $\mathrm{b}$ ) sont les mêmes que celles citées précédemment.

Le choix de $C_{130}$ comme variable indépendante dans l'expression de la biomasse des troncs s'explique par le bon résultat qu'elle permet d'obtenir dans des peuplements simples (équiens et homogènes). II est cependant nécessaire d'utiliser un correctif pour minimiser au mieux l'erreur d'évaluation de la biomasse lors de la transformation du résultat de l'équation logarithmique en données arithmétiques: $Y=10\left(a+b \log (X)+S^{2 / 2}\left(S^{2}=\right.\right.$ variance). Cette erreur $\left(S^{2} / 2\right)$ est estimée à 10-20\% de la biomasse d'un jeune peuplement (Baskerville, 1972). Ce modèle est appliqué pour le calcul de la minéralomasse du tronc (tableau III).

Dans le cas d'une relation curvilinéaire (tableau III), l'évaluation de la biomasse s'obtient selon l'équation :

$$
\begin{aligned}
& M S=\mathrm{a}+\mathrm{b}(X)+\mathrm{c}(X)^{2} \\
& \left(\mathrm{a}, \mathrm{b}, \mathrm{c}=\text { constantes, } X=D_{10} \text { ou } C_{130}\right)
\end{aligned}
$$

Le coefficient de corrélation entre le $D_{10}$ ou $C_{130}$ et les quantités des différents éléments est significatif à $5 \%$, excepté pour le calcium et le manganèse dans le bois des branches. $D_{10}$ n'explique pas toute la variabilité, il est probable que d'autres facteurs peuvent avoir une influence, par exemple le degré de jaunissement des aiguilles.

\section{Choix du tarif le moins biaisé}

Dans certains cas (matière sèche, calculée en fonction de $C_{130}$, biomasse ou encore de la minéralomasse du tronc), on est en présence de 3 relations : polynomiale, linéaire et relation allométrique, qui sont toutes hautement significatives à $1 \%$. Le choix du modèle approprié comme tarif est basé sur la comparaison de la distribution des résidus, méthode la plus efficace pour 
Tableau III. Exemples de modèles de tarifs d'évaluation de la biomasse et de la minéralomasse à partir de la variable dendrométrique $C_{130}$ (Circonférence à $1,30 \mathrm{~m}$ en $\mathrm{cm}$ ).

$\begin{array}{llll}\text { Compartiment Elts ou MS Tarifs } & \text { ET } & R\end{array}$

Aiguilles

Mat sèche

$(\mathrm{T}),(\mathrm{N})$

(Ca), (PCa)

$\mathrm{Ca}$

$\begin{array}{llll}(\mathrm{T}),(\mathrm{N}) & -3778,3+10,09\left(C_{130}\right) & 957 & 0,785^{* * *} \\ (\mathrm{Ca}),(\mathrm{PCa}) & -7751,7+26,09\left(C_{130}\right) & 3118 & 0,765^{* * *}\end{array}$

Bois des branches vivantes

Mat sèche

$(\mathrm{T}),(\mathrm{N})$

(Ca), (PCa)

$\mathrm{Ca}$

$\begin{array}{lll}-26653,6+67,6\left(C_{130}\right) & 0,79 & 0,787^{\star * *} \\ -26401,1+75,7\left(C_{130}\right) & 0,82 & 0,815^{\star * *} \\ -89,03+0,22\left(C_{130}\right) & 0,85 & 0,85^{\star * *} \\ -107,2+0,28\left(C_{130}\right) & 0,85 & 0,853^{\star * *}\end{array}$

Bois du tronc

Mat sèche

(T), (N) $71,92-0,224\left(C_{130}\right)+0,00046\left(C_{130}\right)^{2} \quad 20,1 \quad 0,963^{* * *}$

(Ca), (PCa) $-320,5+0,82\left(C_{130}\right)-0,00017\left(C_{130}\right)^{2} \quad 27,5 \quad 0,949^{* * *}$

Ca $483,93-1,32\left(C_{130}\right)+0,0012\left(C_{130}\right)^{2} \quad 58 \quad 0,859^{* * *}$

$M g-5,37+2,32 \log \left(C_{130}\right) \quad 9,3 \quad 0,871^{* \star *}$

$E T=$ erreur; $R$ : coefficient de corrélation; ${ }^{* * *}=$ significatif à $1 \%$.

apprécier le modèle le moins biaisé (Baskerville, 1972).

\section{Résultats de l'application des tarifs : évaluation de la biomasse et de la minéralomasse}

Les modèles ou tarifs sont alors appliqués aux données de l'inventaire du peuplement. L'effet de la fertilisation sur la biomasse et la minéralomasse peut alors être évalué.

\section{Répartition des éléments} dans le peuplement

La répartition de la minéralomasse dans l'arbre diffère selon l'élément et le compartiment considérés (tableau IV). La quantité d'azote contenue dans les parties les plus jeunes de l'arbre (aiguilles et bois des branches) du peuplement représente $62 \%$ de l'azote total. C'est en fait la partie qui retournera au sol après exploitation du tronc seul. Dans celui-ci, le $\mathrm{K}$ et le Ca représentent chacun $68 \%$, le N $38 \%$, le S $47 \%$, le P $45 \%$ et le $\mathrm{Mg} 67 \%$ de leur valeur totale dans l'arbre. 


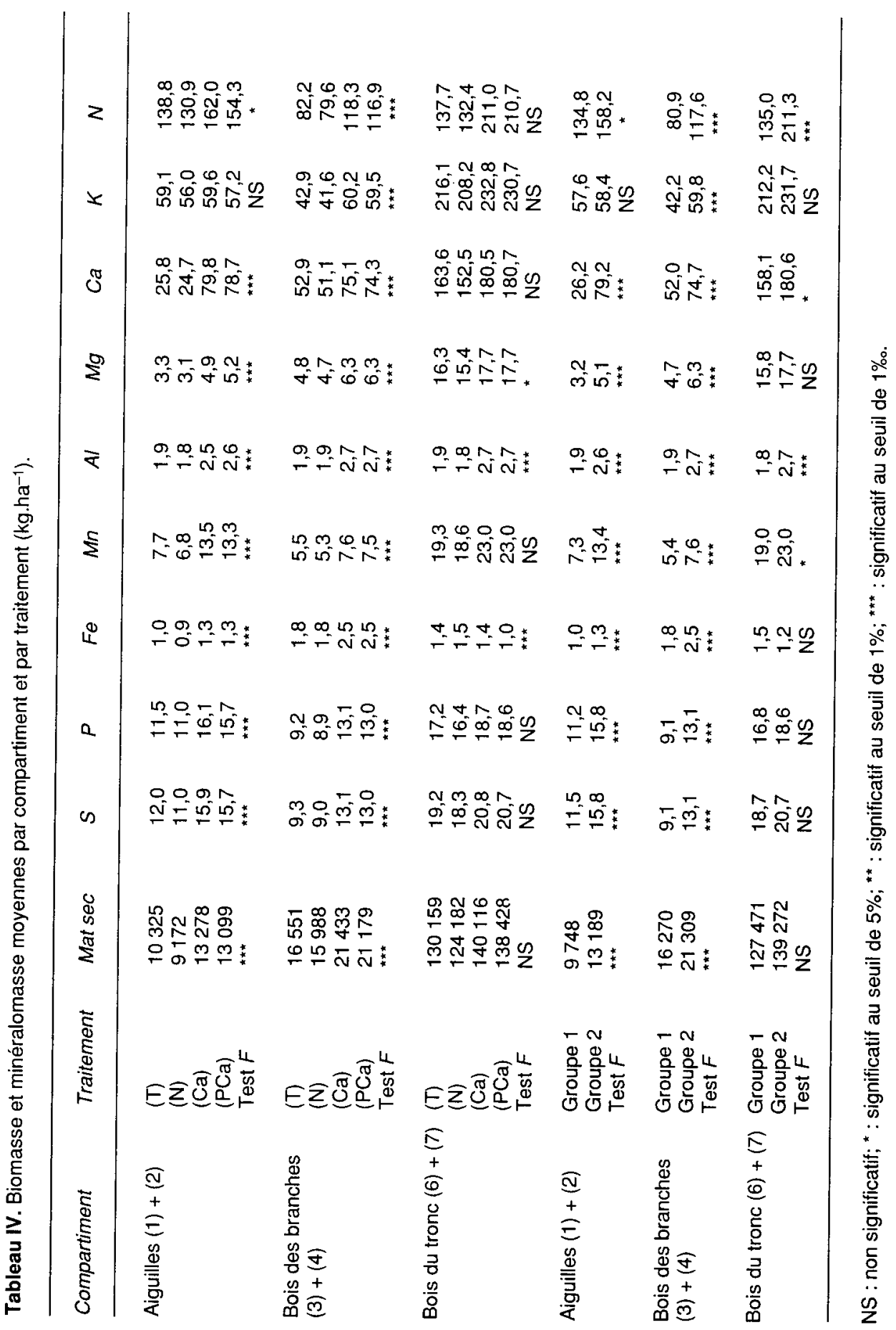


La minéralomasse de la partie épigée de l'arbre offre la succession suivante : $\mathrm{N}>\mathrm{K}>\mathrm{Ca}>\mathrm{S}>\mathrm{P}>\mathrm{Mn}>\mathrm{Mg}>\mathrm{Al}>\mathrm{Fe}$.

\section{Effet des traitements sur la biomasse et la minéralomasse des aiguilles et du buis}

La production en biomasse et l'accumulation de la minéralomasse (tableau IV), montrent 2 groupes de traitements présentant des différences significatives $(T)$ et (N) d'une part, (Ca) et (PCa) d'autre part. À l'intérieur de ces groupes, les traitements ne sont pas significativement différents. L'ammonitrate dans le premier groupe et l'apport de phosphore dans le second groupe n'ont pas eu d'effet hautement significatif sur la biomasse et la minéralomasse. L'apport de calcaire, sous ses différentes formes, a accru les quantités d'éléments minéraux et de matière sèche des aiguilles. La quantité de calcium dans les aiguilles est presque 3 fois plus élevée dans les traitements $(\mathrm{Ca})$ et (PCa) que dans les traitements $(T)$ et $(N)$, il en est de même dans une moindre mesure pour la plupart des éléments, sauf pour le potassium. Ce dernier est le plus mobile dans l'écosystème et peut être absorbé at restitué plusieurs fois en un temps court; il est également peu engagé dans les combinaisons organiques. Sa teneur élevée dans le $(T)$ et le traitement $(N)$ n'apparaît pas automatiquement dans la minéralomasse des aiguilles. Le calcaire a amélioré la nutrition minérale des arbres, ce qui a eu pour conséquence une augmentation de la biomasse et de la minéralomasse des aiguilles du peuplement amendé. L'apport d'azote sous forme d'ammonitrate a eu un effet inverse sur la matière sèche et la quantité des éléments minéraux. Les valeurs sont inférieures à celles du $(T)$, de $11 \%$ pour la matière sèche des aiguilles. Si la teneur en azote a augmenté sensiblement dans les aiguilles par rapport aux autres traitements, sa quantité, par contre, a diminué de $6 \%$. De nombreuses expériences ont montré, sauf exception, que les engrais azotés employés seuls ont plutôt un effet dépressif, surtout sur les résineux (Bonneau, 1986). Delinge et André (1985) ont constaté que l'apport d'azote sous forme d'urée a induit une perte de croissance allant jusqu'à $3,6 \%$, dans une pessière d'âge moyen. L'apport d'ammonitrate a un effet une année après l'épandage, mais n'a plus d'effet sur la croissance en hauteur après 9 ans (Bonneau, 1986). Madgwick et Satoo (1975) ont observé que le sulfate et le phosphate d'ammonium n'ont eu aucune influence sur la biomasse après 4 ans. Miller et Miller (1976) ont par contre constaté, après fertilisation au sulfate d'ammonium, une nette augmentation, de 25$35 \%$, de la masse foliaire sur le pin de Corse âgé de 36 ans. II est certain que l'effet d'un apport d'azote dépend de la disponibilité des autres éléments, mais aussi de l'âge du peuplement fertilisé.

La biomasse et la minéralomasse du bois dans les traitements $(\mathrm{Ca})$ et (PCa) sont plus élevées que dans le $(T)$. Les quantités de matière sèche respectives du tronc et des branches vivantes ont varié par rapport au ( $\mathrm{T}$ ) de $+8 \%$ et $+30 \%$ dans les traitements ( $\mathrm{Ca}$ ) et ( $\mathrm{PCa}$ ), de $-4 \%$ et $-3 \%$ dans le traitement $(N)$. La différence entre le (T) et le traitement (N) n'est pas significative. Les gains en biomasse sont hautement significatifs (à $1 \%$ ou $1 \%$ ) pour les branches vivantes et significatifs à $9 \%$ pour le tronc (tableau IV). II faudrait cependant analyser les 5 derniers cernes pour distinguer un éventuel effet discriminant des traitements sur ce compartiment car la moyenne sur 60 ans dilue cet effet. 


\section{DISCUSSION ET CONCLUSION}

L'analyse minérale qualitative montre les très faibles teneurs moyennes (proches du seuil de carence) en $\mathrm{Mg}$ des aiguilles du (T) et du traitement (N). La teneur en $\mathrm{Ca}$ est aussi faible dans le (T) et (N). L'apport des scories ou de carbonate de calcium pur a amélioré sensiblement la teneur des aiguilles, quelle que soit leur position, en S, P, Ca, Mg (Belkacem, 1990). Cependant, l'effet des traitements est plus net dans les verticilles les plus jeunes, que se soit pour les aiguilles ou pour le bois.

Les faibles teneurs minérales des aiguilles, plus spécialement en $\mathrm{Mg}$, reflètent bien les symptômes de dépérissement signalés sur les arbres des traitements $(T)$ et (N). L'apport de calcium sous ses différentes formes a eu un effet favorable sur la nutrition minérale des arbres sauf pour $K$ et $\mathrm{N}$; cela ne peut s'expliquer, pour $\mathrm{Mg}$ et les autres éléments, que par un effet indirect induit par le $\mathrm{Ca}$ dans le sol. Il est montré par plusieurs auteurs (Toutain et al, 1988; Persson et al, 1989) que le Ca provoque une amélioration de l'ensemble des propriétés physiques, chimiques et biologiques du sol. Cela s'explique par le fait que la faune et la microfaune du sol trouvent des conditions favorables pour décomposer plus efficacement la matière organique et augmenter ainsi la réserve disponible en éléments minéraux. Par contre, la fertilisation azotée, dans ce type de sol qui reçoit déjà annuellement une quantité importante d'azote, évaluée à $50 \mathrm{~kg} \cdot \mathrm{ha}^{-1} \cdot \mathrm{an}^{-1}$ (Nys, 1989) et qui est mal pourvu en $\mathrm{Ca}$ et $\mathrm{Mg}$, ne fait qu'augmenter son acidité par nitrification. Les nitrates entraînent des cations qui sont alors lessivés vers la profondeur.

L'analyse en composantes principales $(A C P)$, réalisée sur chacun des compartiments, ne fait que confirmer les analyses précédentes sur le bois et les aiguilles.
Cette analyse nous montre une séparation nette des groupes de traitements cités antérieurement, par exemple la concentration minérale des aiguilles et du bois des verticilles supérieurs (fig 3). La séparation est moins marquée dans les aiguilles et bois des branches basses et il n'y a aucune différence entre traitements pour le bois du tronc (Belkacem, 1990).

La biomasse et la minéralomasse diffèrent selon le type de fertilisation. Les traitements $(\mathrm{Ca})$ et $(\mathrm{PCa})$ ont induit un accroissement de la biomasse totale de $8-10 \%$ et de la minéralomasse de $11-57 \%$ (fig 4). La fertilisation azotée a, par contre, entraîné une diminution de $4 \%$ pour la biomasse et de $3-8 \%$ pour la minéralomasse. La comparaison entre les 4 traitements montre

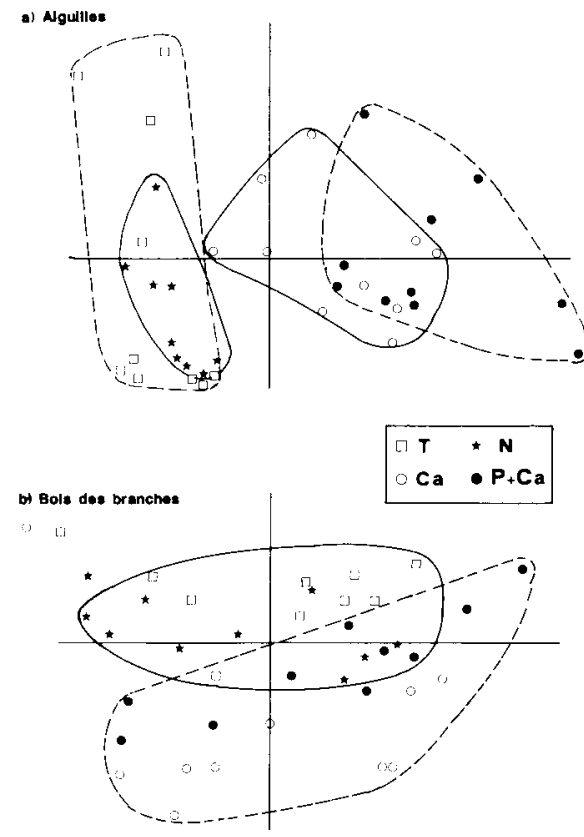

Fig 3. Analyse en composantes principales des données : concentration des aiguilles et du bois des branches des verticilles supérieurs des arbres des différents traitements. 

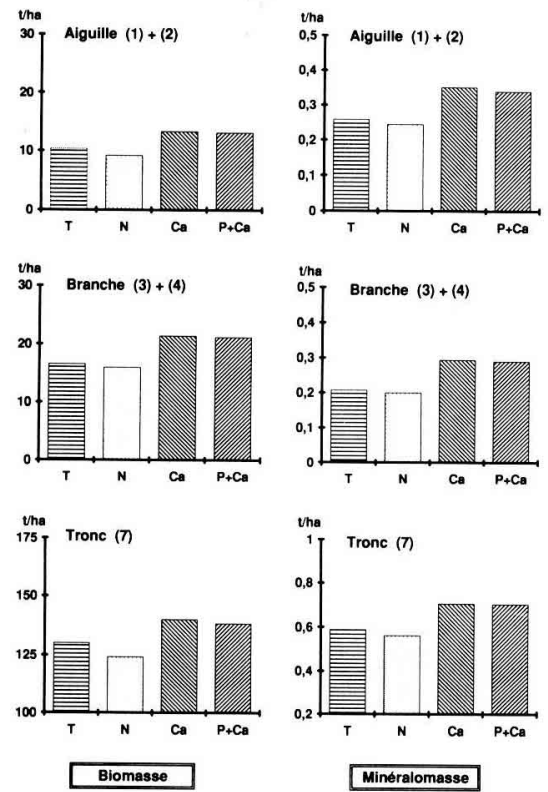

Fig 4. Répartition de la biomasse et de la minéralomasse par traitement dans les aiguilles et les branches des verticilles supérieurs et dans la partie inférieure du tronc.

que la perte de la biomasse d'aiguilles dans le traitement ( $N$ ) par rapport au (T) est de $11 \%$. Parallèlement, un gain de $28 \%$ de la masse d'aiguilles est observé dans le traitement (Ca) et de $29 \%$ dans le traitement (PCa).

Les rapports entre les quantités moyennes du ( $\mathrm{Ca}$ ) et ( $\mathrm{PCa}$ ) et celles du ( $T$ ) sont résumés dans le tableau $V$. Ils permettent de faire le parallélisme entre le gain en biomasse et celui en minéralomasse pour les compartiments des aiguilles $[(1)+(2)]$ et des branches (bois + écorce $)[(3)+(4)]$ formés pendant la période post fertilisation. Les rapports des biomasses $(1,28)$ calculés entre les traitements témoins et amendés sont identiques pour les compartiments des aiguilles et des branches. Les gains en minéralomasse sont supérieurs à ceux de la biomasse pour le compartiment des branches, sauf pour le Mg. Pour les aiguilles, les rapports pour le $\mathrm{Mg}$ et le $\mathrm{P}$ sont supérieurs à celui de la biomasse; par contre ils sont inférieurs pour l'azote et surtout pour le $K$. Pour ce dernier élément il n'y a pas de prélèvement supplémentaire par rapport au témoin.

L'azote et le $\mathrm{K}$ absorbés en supplément dans les traitements ( $\mathrm{Ca}$ ) ou (PCa) sont essentiellement utilisés pour élaborer du bois et de l'écorce; le P l'est également. Le Mg est, par contre, plus incorporé dans les aiguilles. Le calcul séparé pour les traitements (Ca) et (PCa) donne des rapports équivalents signifiant que l'ajout de $P$ est sans relation avec le gain de biomasse. II y

Tableau V. Rapports moyens entre les biomasses ou les quantités d'élément des traitements (Ca) et (PCa) et du traitement ( $T$ ).

$\begin{array}{llllll}\frac{[\mathrm{Q}](\mathrm{Ca})+(\mathrm{PCa})}{2 \times[Q](T)} & \text { Biomasse } & M g & N & K & P\end{array}$

$\begin{array}{llllll}\text { Aiguille (a) } & 1,28 & 1,53 & 1,14 & 0,99 & 1,50 \\ \text { Branche (b) } & 1,29 & 1,31 & 1,43 & 1,39 & 1,42 \\ \text { (a) }+ \text { (b) } & 1,28 & 1,40 & 1,25 & 1,17 & 1,40\end{array}$

(a) $=$ Compartiments $(1)+(2) ;(b)=$ compartiments $(3)+(4)$. 
aurait donc un effet du (Ca) sur la dynamique de l'absorption par le système racinaire.

L'analyse de ces rapports permet d'élaborer quelques hypothèses qu'il nous faudra vérifier. Si l'on estime que l'azote n'est pas un élément limitant, compte tenu des apports élevés dans cette région, on peut formuler l'hypothèse que l'effet biomasse ne reflète pas un effet sur la minéralisation de l'azote mais plutôt un effet racinaire (sur l'absorption de l'azote). Pour le $\mathrm{Mg}$, bien que cet élément n'ait pas été apporté, l'effet est supérieur principalement pour le compartiment des aiguilles. On peut estimer qu'il est dû à un effet "minéralisation" ou bien encore en partie à un effet "échange" avec le calcium. Pour le $\mathrm{K}$, il semble $y$ avoir antagonisme avec le calcium (déplacement par échange) et enfin pour le phosphore, une amélioration du dynanisme de son absorption racinaire.

L'évaluation de la minéralomasse (tableau IV) nous a permis de mesurer l'immobilisation du peuplement depuis 60 ans et celle-ci est représentée par compartiment dans la figure 4 . Cette minéralomasse ne représente que celle de la partie épigée du peuplement. II faudra distinguer entre ce qui retournera au sol (aiguilles, branches) et ce qui sera définitivement perdu par l'écosystème (tronc), ce dont on doit tenir compte pour une fertilisation à la prochaine révolution.

La comparaison des présents résultats avec 2 peuplements adultes d'épicéa commun, est résumé dans le tableau VI. La biomasse moyenne annuelle est plus élevée aux Champs-Bernard, peuplement voisin de celui de cette étude mais ncn dépérissant, qu'à la Croix-Scaille (Ardennes). Après apports du calcaire, les valeurs se rapprochent de celles du site d'Aubure (Vosges) où le peuplement présente cependant des signes de dépérissement (Le
Goaster, 1989). Les accroissements moyens annuels de minéralomasse en $\mathrm{N}$ et en $\mathrm{P}$ sont comparables dans le peuplement du Champs-Bernard et les peuplements amendés de la présente étude. Les peuplements $(T)$ et fertilisé $(N)$ ont une minéralomasse en $P$ et $N$ voisine de celle du peuplement d'Aubure. Pour le Mg, les valeurs sont plus faibles quel que soit le traitement à la Croix-Scaille, ce qui confirme la pauvreté de la station en cet élément. Pour le calcium, les traitements (Ca) et $(\mathrm{PCa})$ aboutissent à des valeurs voisines des 2 autres sites.

Les sites d'Aubure et de la Croix-Scaille sont relativement plus pauvres en $\mathrm{Mg}$ et en $\mathrm{Ca}$ que celui du Champs-Bernard et se caractérisent par des symptômes de dépérissement lesquels disparaissent par apports de calcaire à la Croix-Scaille. Les arbres du Champs-Bernard restent sains, du fait probablement de la richesse minérale du sol, lequel contient environ 2 fois plus de réserve d'éléments échangeables qu'à la Croix-Scaille (Nys, 1989).

Les apports par la pluie et les dépôts secs d'éléments acidifiants azotés et soufrés sont relativement élevés dans ce site. Il y a eu un déséquilibre nutritionnel (N/Mg, $\mathrm{N} / \mathrm{Ca}$ et $\mathrm{S} / \mathrm{N}$ ) provoqué par une désaturation lente du sol, du fait d'un lessivage important des éléments en profondeur, en plus des prélèvements par la végétation. Dans de pareilles conditions et au vu de ces résultats, les apports d'amendements s'avèrent utiles, voir indispensables, afin de remédier au déséquilibre de ce milieu. Les doses et formes des apports restent cependant à préciser.

Nous pouvons retenir de cette étude le rôle important du Ca plus probablement par une action indirecte sur l'amélioration des conditions de décomposition de la matière organique des sols acides, comme l'ont signalé plusieurs auteurs, que par une 


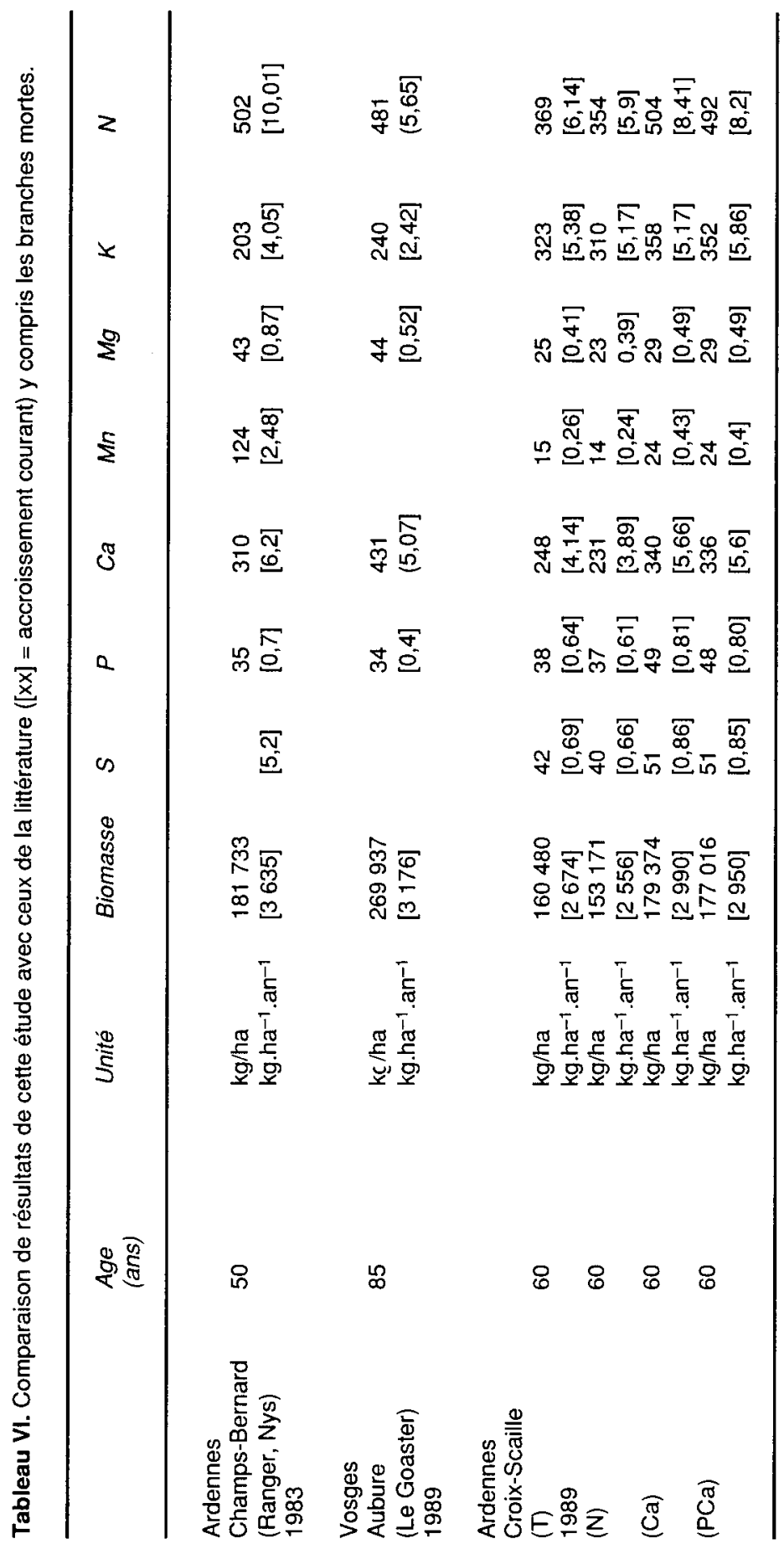


action directe sur la nutrition. La levée de carence magnésienne est liée au rôle indirect du calcaire. Exacerbé par les apports atmosphériques acides qui ne font qu'amenuiser les réserves cationiques du sol, le déséquilibre entre le $\mathrm{Mg}$ et le $\mathrm{N}$ s'accroît dans ces peuplements dépérissants du fait d'une grande disponibilité en azote dans le sol (Oren et al, 1988). Noids nous associons aux nombreux auteurs qui écrivent que la cause de la baisse de vigueur des arbres reflète plutôt une dysharmonie nutritionnelle que l'effet d'un seul élément.

L'amendement calcique peut donc permettre d'accroître la production de biomasse forestière et même contribuer dans certains cas à améliorer l'état sanitaire des arbres. Cette correction du niveau de fertilité de la station doit cependant être menée en respectant un certain équilibre de fonctionnement de l'écosystème forêt.

\section{RÉFÉRENCES}

Andersson $F$ (1989) Effects of liming on tree growth, a synthesis of Swedish-ecosystem experiments, IUFRO Symp Manag Nutr For Stress. Freiburg, W Germany, sept 18-21

Baskerville GL (1972) Use of logarithmic regression in the estimation of plant biomass. Can J For 2, 49-53

Becquer T, Merlet D, Boudot JP, R uiller J, Gras $F(1990)$ Nitrification and nitrate uptake: leaching balance in a declined forest ecosystem in eastern France. Plant Soil 125, 95-107

Belkacem S (1990) Effet d'un amendement calcaire et d'une fertilisation azotée sur la redistribution des éléments minéraux dans le sol et dans la biomasse forestière. DEA, Fac Nancy I, $80 \mathrm{p}$

Bonneau M (1986) Diagnostic foliaire. Polycopié, ENGREF, Centre de Nancy

Bonneau M (1988) Le diagnostique foliaire. Rev For Fr XL, $\mathrm{n}^{\circ} \mathrm{sp}, 19-28$

Bonneau M, Landmann G (1988) Le dépérissement des forêts en Europe. Recherche 205, 1542-1556
Clément A (1977) Point 77 sur certaines analyses foliaires. Brochure interne : station de recherche sur les sols forestiers et la fertilisation, INRA Nancy

Dagnélie $P$ (1974) Analyse statistique à plusieurs variables. Presses Agron Gembloux, Wander SA

Dagnélie P, Palm R, Rondeux J, Thill A (1988) Tables de productions relatives à l'épicéa commun. Presses Agron Gembloux, 120 p

Delinge J, André $P$ (1985) Essais de fertilisation de pessière d'âge moyen. Bull Soc Roy Belg $6,247-258$

Dubs A (1988) Étude par les techniques de lysjmétrie et de résines échangeuses de cations, du fonctionnement géochimique moyen d'un sol brun acide forestier sous épicéa et de la modification de celui-ci par un traitement de fertilisation. DEA de pédologie, Univ Pierre et Marie Curie, Paris VI, $51 \mathrm{p}$

Hoehne $H$ (1964) Untersuchungen über die jahreszeitlichen Veränderungen des Gewicht und Elementgehalt. Arch Forstwes 13, 7, 747-774

Landmann G, Bonneau M, Adrian M (1987) Le dépérissement du sapin pectiné et de l'épicéa commun dans le massif Vosgien, est-il en relation avec l'état nutritionnel des peuplements ? Rev For Fr XXXIX, 1, 5-11

Le Goaster $S$ (1989) Influence de la croissance d'un peuplement sur le prélèvement en éléments minéraux, relation avec le dépérissement. Mémoire de $3^{\theta}$ année ingénieur, ENITEF, $83 p$

Lemoine B, Ranger R, Gelpe J (1990) Interprétation et modélisation de la distribution des éléments nutritifs dans les différents compartiments de la biomasse d'un jeune peuplement de pin maritime. Ann Sci For 47, 101115

Magdwick HAl, Satoo T (1975) Estimating the above-ground weights of tree stands. Ecology $56,1446-1450$

Miller HG, Miller JD (1976) Effects of nitrogen supply on net primary production in Corsican pine. In: Proc Aust For Nutr Workshop, Caminra, Australia, 10-14 August, 187-199

Nys $C$ (1989) Fertilisation, dépérissement et production de l'épicéa commun (Picea abies) dans les Ardennes. Rev For Fr XLI, 4, 336 . 347 
Nys C, Ranger J (1988) Influence d'une substitution d'espèce sur le fonctionnement biogéochimique de l'écosystème forêt : l'exemple du cycle du soufre. Ann Sci For 45(3), 169188

Nys C, Ranger J, Ranger D (1983) Étude comparative de 2 écosystèmes forestiers feuillus et résineux des Ardennes primaires françaises. Ann Sci For 40, 1, 41-66

Oren R, Schulze ED, Meyer J, Schneider BU, Heilmeier $H$ (1988) Performance of two Picea abies $\mathrm{L}$ Karst stands at differents stages of decline. Oecologia 77, 25-35, 151162, 163-173

Pardé J (1980) Forest biomass review. CAB, 41-81, 431-362

Persson T, Wiren A, Anderssons S (1989) Effects of acidification and liming on carbon and nitrogen mineralization and soil organisms in mor humus. Water Air Soil Pollut 45, 77-96

Ranger R (1981) Étude de la minéralomasse et du cycle biologique dans deux peuplements de pin Laricio de Corse, dont l'un a été fertilisé à la plantation. Ann Sci For 38, 1, 127151
Ranger J, Barneoud C, Nys C (1988) Production ligneuse et rétention d'élément nutritifs par des taillis à courte rotation de peuplier «Rochester» : effet de la densité d'ensouchement. Oecol Plant 9, 3, 245-269

Rapp M (1978) Le cycle biogéochimique dans un bois de pin d'Alep. In: Ecologie forestière, la forêt : son climat, son sol, ses arbres, sa faune (P Pesson, ed), Gauthier-Villars, Paris

Riedacker A (1968) Méthode d'estimation de la biomasse d'un arbre. DEA Biol Vég. Fac Orsay, $31 \mathrm{p}$

Satoo T, Madwick HAI (1982) Forest biomass, forestry sciences. Nijhoff $M$, Junk WJ Publ La Haye

Toutain F, Diagne A, Le Tacon F (1988) Possibilités de modification du type d'humus et d'amélioration de la fertilité des sols à moyen terme en hêtraie par apport d'éléments minéraux. Rev For Fr XL, 2, 99-107

Wells CG, Metz LS (1963) Variation of mineral nutrient content of Loblolly pine needles with season, age, soil and position on the crown. Soil Sci Am Proc 27, 90-93 